\title{
Prévalence de la douleur chronique et des limitations fonction- nelles qui lui sont associées au Canada entre 1994 et 2008
}

M. L. Reitsma, M. Sc. (1); J. E. Tranmer, Ph. D. (1); D. M. Buchanan, Ph. D. (1); E. G. Vandenkerkhof, Ph. D. - santé publique (1,2)

Cet article a fait l'objet d'une évaluation par les pairs.

\section{Résumé}

Introduction : On observe actuellement une absence de concordance entre la prévalence estimative de la douleur chronique au Canada et dans le monde. Nous souhaitions donc dans le cadre de cette étude déterminer la prévalence de la douleur chronique selon le sexe et l'âge de même que la prévalence des limitations fonctionnelles associées à la douleur chronique selon le sexe, au Canada, pour la période 1994-2008.

Méthodologie : En utilisant les données extraites de sept cycles transversaux de l'Enquête nationale sur la santé de la population et de l'Enquête sur la santé dans les collectivités canadiennes, nous avons défini deux résultats catégoriels, à savoir la douleur chronique et les limitations fonctionnelles associées à la douleur chronique.

Résultats : La prévalence de la douleur chronique variait entre 15,1 \% (1996-1997) et $18,9 \%$ (1994-1995); elle était supérieure chez les femmes (entre 16,5\% et 21,5\%) ainsi que chez les Canadiens les plus âgés, c'est-à-dire chez les membres du groupe d'âge " 65 ans et plus » (entre 23,9\% et 31,3\%). La plus forte prévalence de douleur chronique (entre $26,0 \%$ et $34,2 \%$ ) était observée pour tous les cycles chez les femmes de 65 ans et plus. Finalement, la majorité des adultes canadiens affirmant souffrir de douleur chronique signalaient aussi que cette douleur empêchait la réalisation d'au moins quelques activités (de 11,4\% à 13,3\% de la population totale).

Conclusion : Les résultats de cette étude populationnelle concordent avec les estimations internationales et confirment que la douleur chronique est bien présente au Canada et qu'elle affecte les activités quotidiennes des personnes touchées. Ils mettent aussi en évidence la nécessité de procéder à de plus amples études en employant une définition plus précise de la douleur et des limitations fonctionnelles qui lui sont associées.

Mots-clés : douleur chronique, prévalence, facteurs sociodémographiques, population générale, limitation des activités

\section{Introduction}

Environ 17 \% des Canadiens - 3,9 millions d'individus de plus de 15 ans - ont déclaré souffrir de douleur chronique ou d'un certain degré de malaise ${ }^{1}$. La douleur chronique a une incidence sur la qualité de vie, y compris sur le volet social et familial, ainsi que sur la capacité à travailler².
En 2010, la Chronic Pain Association of Canada a indiqué que "selon les estimations, le coût annuel associé à la douleur chronique, si on considère les frais médicaux et les pertes de revenu et de productivité, mais non les coûts sociaux, serait supérieur à 10 milliards de dollars $»^{3}$.
La prévalence des douleurs chroniques non spécifiques au sein de la population générale atteindrait $55 \%{ }^{4,5}$. Des études canadiennes ont quant à elles conclu à une prévalence estimative de la douleur chronique se situant entre $11 \%$ et $44 \%^{1,2,6-13}$. Ces études étaient fondées sur des périodes de 3 à 6 mois $^{2,6,7,12}$ ou sur une définition de la douleur la qualifiant d'habituelle ou de

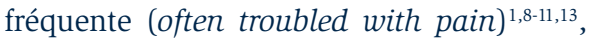
et les estimations de la douleur fondées sur une période plus longue (c.-à-d. douleur habituelle ou persistante) ont donné lieu à des estimations plus faibles de la prévalence ${ }^{1,8-11,13}$. De toutes les études canadiennes, seulement cinq étaient de vastes études en population ${ }^{1,9-11,13}$, et, parmi celles-ci, trois étaient fondées sur des données antérieures issues du cycle 1996-1997 de l'Enquête nationale sur la santé de la population (ENSP) $)^{9,11,13}$.

Les estimations canadiennes aussi bien qu'internationales de la prévalence varient selon l'âge et le sexe, une prévalence supérieure étant observée chez les femmes ${ }^{2,5,6-10,12,14-18}$ ainsi que chez les personnes âgées ${ }^{2,5,8-11,14,15,17-20}$. Certaines études canadiennes analysant la prévalence de la douleur chronique selon le sexe et l'âge n'étaient pas représentatives de la population générale : en effet, l'échantillon de l'une d'entre elles n'était représentatif que de sept comtés du Sud-Est de l'Ontario ${ }^{12}$, tandis que, dans un autre cas, il ne l'était que des habitants d'une ville située près de Toronto ${ }^{8}$. Toutefois, les résultats d'études transversales en population fondées sur des données antérieures (1996-1997) et d'études de moindre envergure donnent à penser qu'au Canada, les femmes et les personnes âgées font plus souvent état

Rattachement des auteurs :

1. School of Nursing, Queen's University, Kingston (Ontario), Canada

2. Department of Anesthesiology, Queen's University, Kingston (Ontario), Canada

Correspondance : Elizabeth VanDenKerkhof, Department of Anesthesiology \& Perioperative Medicine, Queen's University, 76 Stuart St., Kingston (Ontario) K7L 2V7;

tél. : 613-549-6666 poste 3964; téléc. : 613-548-1375; courriel : ev5@queensu.ca 
de douleur chronique. Bien que des études se soient déjà penchées sur les entraves aux activités quotidiennes imposées par la douleur ${ }^{1,2,7,8,10,12,13}$, aucune étude n'a examiné cet aspect chez les Canadiens au fil des années.

Cette étude visait à examiner l'évolution dans le temps de la prévalence globale de la douleur chronique et des limitations fonctionnelles qui lui sont associées, au Canada, sans tenir compte des facteurs associés à la douleur. Plus précisément, les objectifs fixés étaient 1) d'analyser la prévalence de la douleur chronique chez les Canadiens entre 1994 et 2008, 2) de décrire les variations de la prévalence de la douleur chronique selon le sexe et l'âge et 3 ) de décrire les variations selon le sexe de la prévalence des limitations fonctionnelles associées à la douleur chronique.

\section{Méthodologie}

\section{Questionnaire et collecte des données}

À l'aide de données extraites de sept cycles transversaux du volet sur les ménages de l'ENSP (1994-1995, 1996-1997, 1998-1999) et de l'Enquête sur la santé dans les collectivités canadiennes (ESCC) (2000-2001, 2003, 2005 et 2007-2008), nous avons étudié l'évolution dans le temps de la prévalence de la douleur chronique au Canada. Ces enquêtes font appel à des entrevues structurées réalisées en personne et par téléphone pour recueillir des renseignements sur l'état de santé et les déterminants de la santé des participants de même que sur leur utilisation des services de santé et des services médicaux ${ }^{21,22}$.

L'ENSP a été réalisée pour la première fois en 1994 en tant qu'enquête transversale et longitudinale; en 2000-2001, sa composante transversale a été intégrée à l'ESCC, qui est toujours menée par Statistique Canada ${ }^{21}$. L'ENSP et l'ESCC ont été réalisées aux deux ans jusqu'en 2007, année où l'ESCC est devenue une enquête annuelle, mais les résultats combinés pour 2007-2008 ont aussi été publiés ${ }^{23}$. Les deux enquêtes ont été élaborées par des spécialistes de Santé Canada, de Statistique Canada et des ministères de la Santé provinciaux de même que par des chercheurs universitaires dans les domaines pertinents, puis les questionnaires ont été approuvés par des comités consultatifs et d'experts. Il est possible d'obtenir de plus amples renseignements sur le plan de sondage de l'ENSP et de l'ESCC auprès d'autres sources ${ }^{21,22}$.

\section{Population et échantillon}

Notre analyse portait sur les participants à l'ENSP âgés de 25 ans et plus et les participants à l'ESCC âgés de 20 ans et plus. Cette différence est attribuable au fait que les tableaux de la variabilité d'échantillonnage n'étaient pas présentés selon les mêmes groupes d'âge par Statistique Canada $(12-24,25-44,45-64$ et 65 ans et plus pour l'ENSP et 12-19, 20-29, 30-34, 45-64 et 65 ans et plus pour l'ESCC). Même si certaines études antérieures ont inclus des participants âgés de seulement 15 ans $\mathrm{s}^{11,14,15,19}$, nous avons fixé la limite inférieure à 20 ans afin d'éviter de regrouper et de comparer des participants adultes et des adolescents. Comme deux études prospectives ${ }^{24,25}$ et une étude fondée sur les données de l'ENSP ${ }^{13}$ ont aussi ciblé les participants qui étaient âgés de 25 ans et plus au début de l'étude, nous serons en mesure de comparer nos résultats à ceux présentés dans la littérature.

En 1994-1995, 1996-1997 et 1998-1999, l'échantillon du volet sur les ménages de l'ENSP était formé d'habitants des 10 provinces canadiennes et comprenait respectivement 17 626, 81804 et 17244 participants ${ }^{26-28}$. Il avait été constitué à l'aide d'une stratégie d'échantillonnage double par grappes et par logements ${ }^{21}$. Les ESCC 2000-2001, 2003, 2005 et 2007-2008 nécessitaient pour leur part la participation annuelle de 65000 personnes provenant de 121 régions sanitaires réparties dans toutes les provinces et territoires ${ }^{22}$. Les échantillons comprenaient 130827 participants en 2000-2001, 134072 en 2003, 132947 en 2005 et 131061 en $2007-2008^{23,29-31}$. Le recensement le plus récent était utilisé pour déterminer l'échantillon et permettait de tenir compte des décès, naissances et migrations estimatives récents; ainsi, au besoin, des modifications étaient apportées aux régions sanitaires à l'étude en fonction du dernier recensement ${ }^{23,26-31}$. De plus, après pondération correcte de leurs résultats,
l'ENSP et l'ESCC sont considérées comme représentatives de la population ciblée, y compris des provinces et territoires concernés par l'échantillonnage $\mathrm{e}^{23,26-31}$. Les volets transversaux sur les ménages de l'ENSP comme de l'ESCC excluaient les Canadiens habitant en établissement, dans les réserves et dans certaines régions éloignées de même que les membres à temps plein des Forces canadiennes ${ }^{21,22}$. Le taux de réponse était supérieur à $77,6 \%$ pour tous les cycles concernés par la présente étude.

\section{Variables}

\section{Variable de résultat : douleur chronique et limitations fonctionnelles associées à la douleur chronique}

Notre définition de la douleur chronique était fondée sur la question suivante : "Habituellement, êtes-vous sans douleur ou inconfort? $»^{26-30,32,33}$. Les participants ayant répondu " non » à cette question étaient considérés comme atteints de douleur chronique. On demandait ensuite à ces personnes combien d'activités leurs douleurs ou malaises les empêchaient de faire en choisissant l'option " aucune ", " quelques-unes », "plusieurs » ou «la plupart $»^{26-30,32,33}$. Cette définition, employée dans plusieurs études, est reconnue comme une mesure valide de la prévalence de la douleur chronique dans la population générale ${ }^{9,13}$.

\section{Variables indépendantes : âge et sexe}

Nous avons évalué la présence de « douleur habituelle » selon le sexe et l'âge, ainsi que le nombre d'activités dont la réalisation était empêchée en raison de cette douleur selon le sexe. Les participants ont été regroupés selon l'âge en fonction des tableaux fournis par Statistique Canada (25-44, 45-64 et 65 ans et plus pour l'ENSP et 20-44 [20-29 et 30-44], 45-64 et 65 ans et plus pour l'ESCC).

\section{Analyse des données}

Nous avons analysé les données extraites de chacun des cycles de l'ENSP et de l'ESCC séparément à l'aide du logiciel SPSS, version 16.0 (IBM). Pour chaque test statistique, l'échantillon a été pondéré selon la population canadienne à l'aide de 
la variable appropriée, selon le cycle ${ }^{23,26-31}$. Les résultats relatifs à la population canadienne ont été présentés selon le sexe pour chaque cycle à l'aide d'effectifs et de pourcentages. Les différences significatives de la prévalence estimative et des limitations fonctionnelles entre les groupes ont été relatives aux groupes contenant un échandéterminées à l'aide d'intervalles de con- tillon d'au moins 30 participants ont été fiance à $95 \%$. Les poids d'échantillonnage diffusées, conformément aux directives ont été appliqués à toutes les estimations de Statistique Canada en la matière ${ }^{26,29}$. afin de permettre la généralisation à la De plus, nous avons comparé toutes les population canadienne. Seules les données données présentées dans les tableaux de

\section{FIGURE 1}

Prévalence brute de la douleur chronique dans la population canadienne selon les données transversales de l'Enquête nationale sur la santé de la population et de l'Enquête sur la santé dans les collectivités canadiennes, selon le sexe

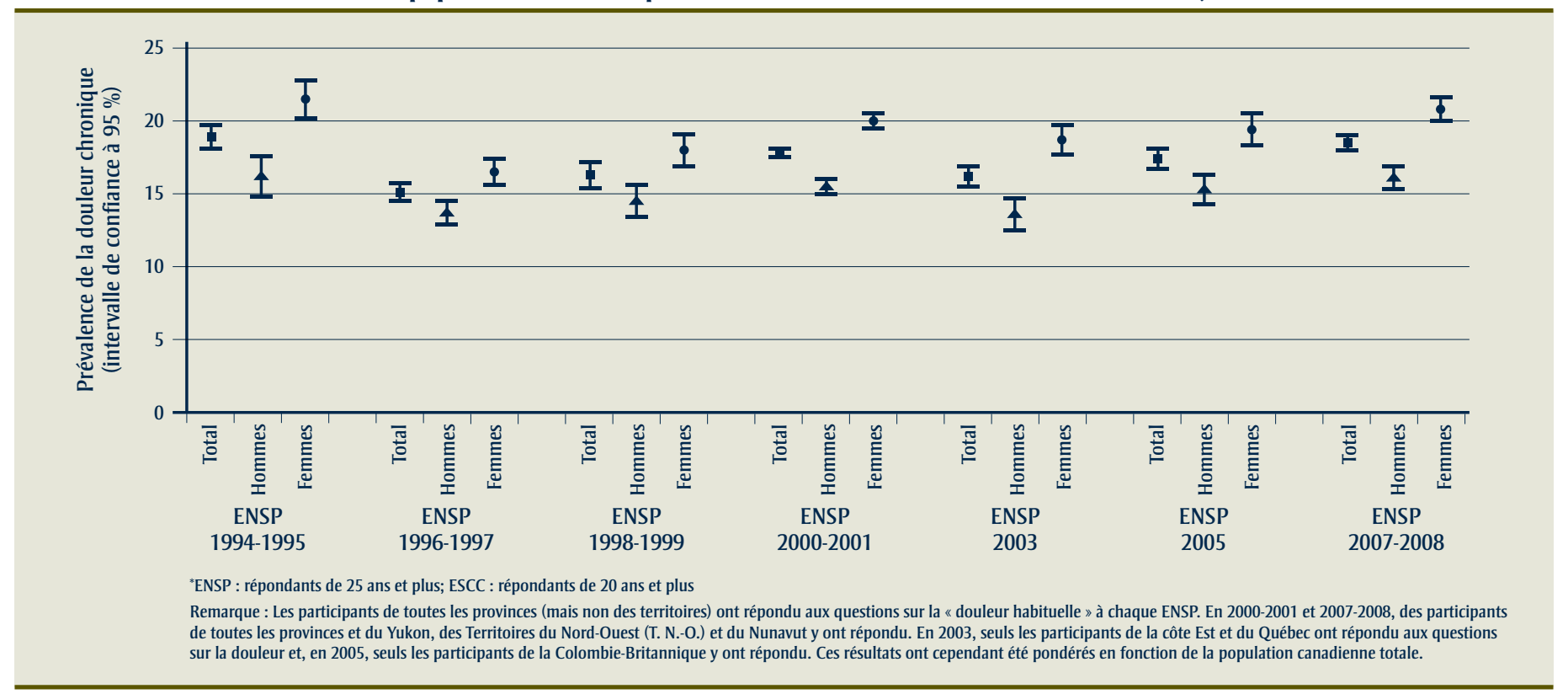

\section{FIGURE 2}

Prévalence brute de la douleur chronique dans la population canadienne selon les données transversales de l'Enquête nationale sur la santé de la population et de l'Enquête sur la santé dans les collectivités canadiennes, selon l'âge

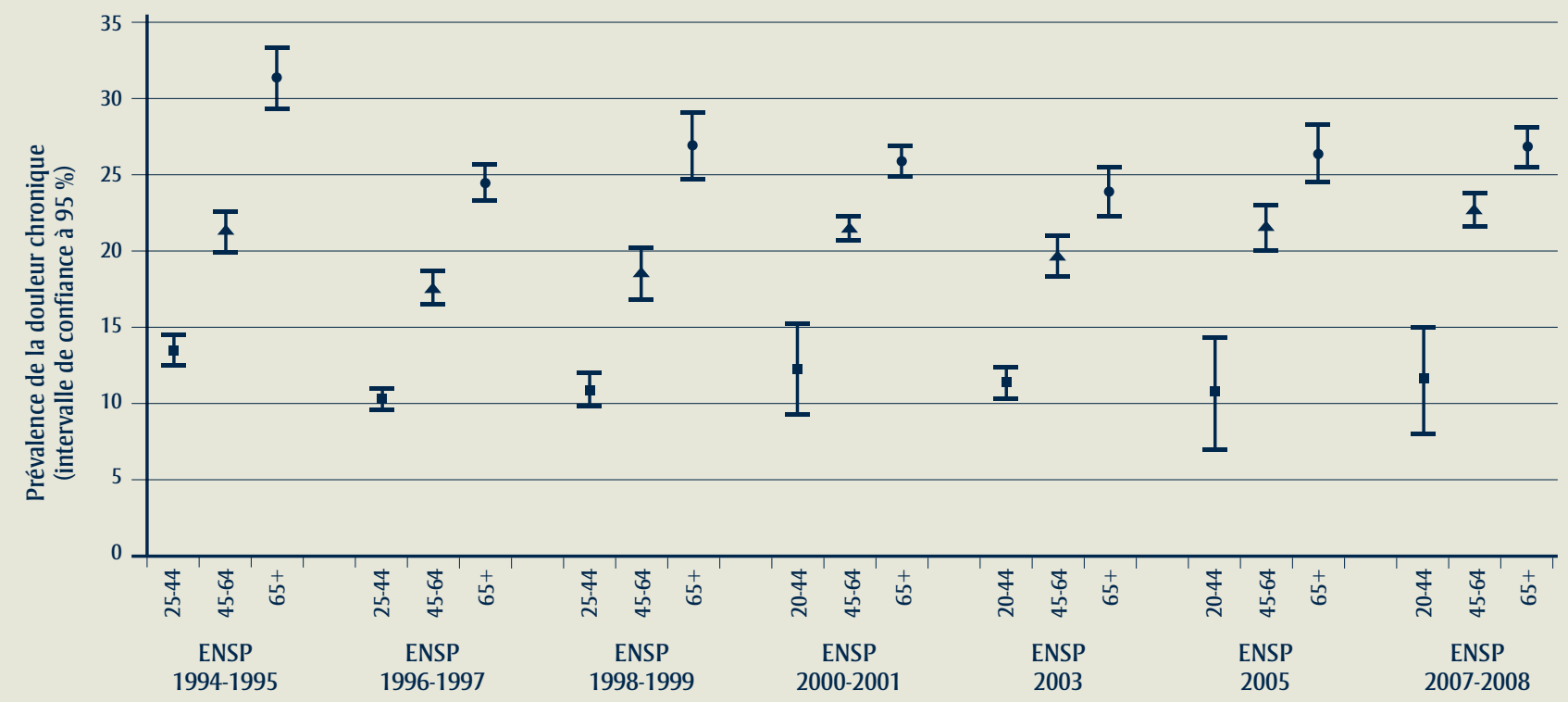

Remarque : Les participants de toutes les provinces (mais non des territoires) ont répondu aux questions sur la « douleur habituelle » à chaque ENSP. En 2000-2001 et 2007-2008, des participants de toutes les provinces et du Yukon, des Territoires du Nord-Ouest (T. N.-O.) et du Nunavut y ont répondu. En 2003, seuls les participants de la côte Est et du Québec ont répondu aux questions sur la douleur et, en 2005, seuls les participants de la Colombie-Britannique y ont répondu. Ces résultats ont cependant été pondérés en fonction de la population canadienne totale. 
FIGURE 3

Prévalence brute de la douleur chronique chez les Canadiens selon les données transversales de l'Enquête nationale sur la santé de la population et de l'Enquête sur la santé dans les collectivités canadiennes, selon l'âge

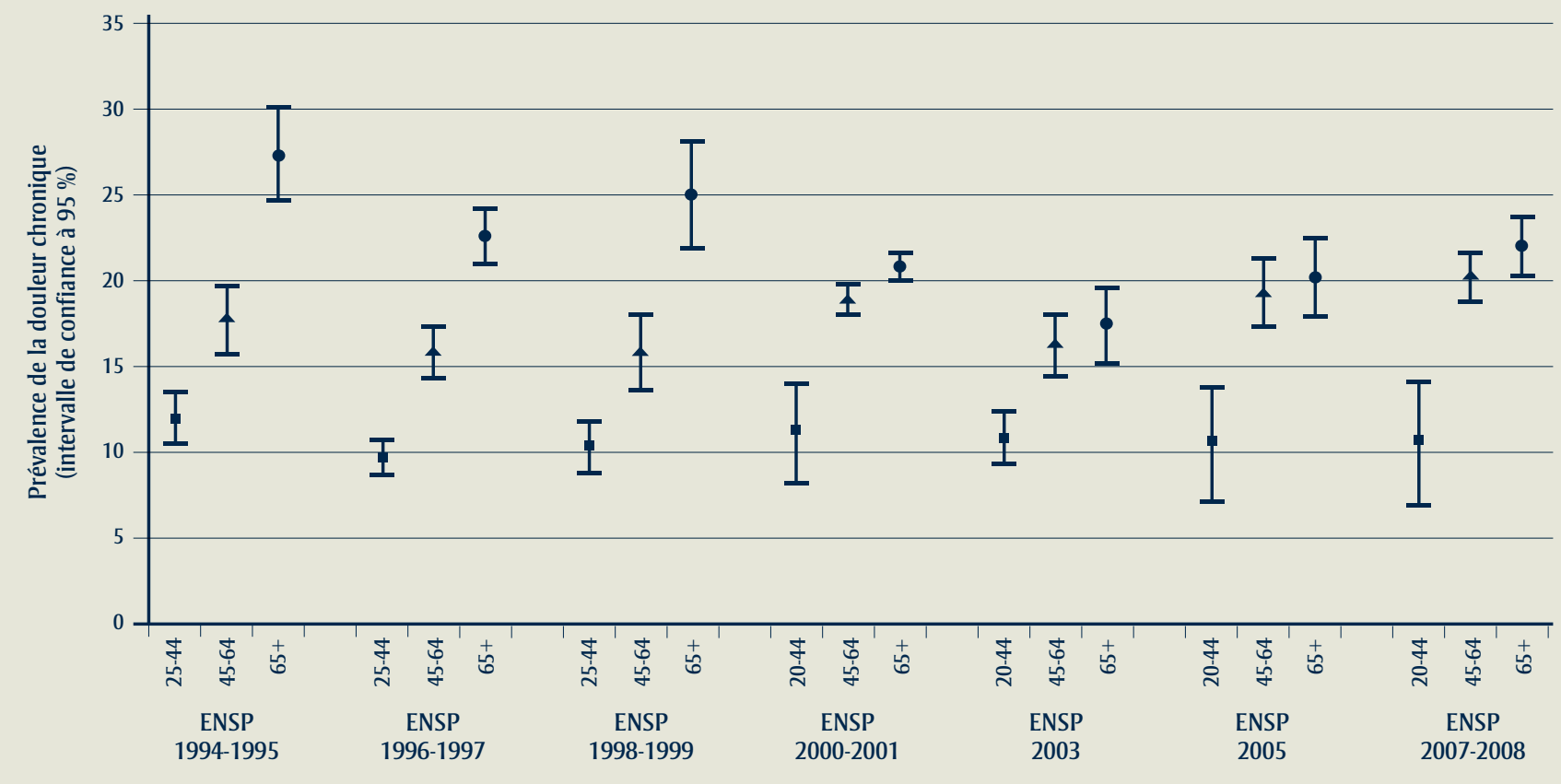

Remarque : Les participants de toutes les provinces (mais non des territoires) ont répondu aux questions sur la « douleur habituelle » à chaque ENSP. En 2000-2001 et 2007-2008, des participants de toutes les provinces et du Yukon, des Territoires du Nord-Ouest (T. N.-O.) et du Nunavut y ont répondu. En 2003, seuls les participants de la côte Est et du Québec ont répondu aux questions sur la douleur et, en 2005, seuls les participants de la Colombie-Britannique y ont répondu. Ces résultats ont cependant été pondérés en fonction de la population canadienne totale.

variabilité d'échantillonnage de Statistique Canada afin de déterminer si la fréquence par cellule pour une variable donnée était suffisante pour éviter qu'un répondant puisse être identifié : si le coefficient de variation se situait entre 0,0 et 16,5 , la diffusion était autorisée; s'il se situait entre 16,6 et 33,3 , la donnée était considérée comme marginale et sa diffusion était autorisée si elle était accompagnée d'une mise en garde (dans l'ENSP, les coefficients de variation se situant entre 25,1 et 33,3 ne pouvaient être diffusés qu'en présence de la variance exacte) et, s'il était supérieur à 33,3 , la diffusion n'était pas autorisée ${ }^{26,29}$. Les intervalles de confiance ont été obtenus à partir des tableaux de la variabilité d'échantillonnage. Dans le cas de l'ESCC 2003, les données estimatives ont été déterminées à l'aide d'un fichier de macros de sous-échantillonnage disponible au Research Data Centre de l'Université Queen's; nous avons aussi appliqué la méthode bootstrap et déterminé les intervalles de confiance à l'aide du logiciel STATA, Data Analysis and Statistical
Software, version 11.0 (StataCorp LP). La méthode bootstrap permet l'obtention de données estimatives sur l'erreur type et d'intervalles de confiance fiables pour de nombreuses estimations, y compris pour les moyennes et proportions ${ }^{34}$. Nous avons soumis cinq cents échantillons à chaque analyse afin de nous assurer que les résultats n'étaient pas seulement significatifs en raison de la grande taille des échantillons.

Le Health Sciences and Affiliated Teaching Hospitals Research Ethics Board de l'Université Queen's a procédé à l'examen de cette méthode d'analyse et l'a approuvée.

\section{Résultats}

\section{Population}

Le ratio hommes/femmes était semblable d'une année et d'une province à l'autre, les femmes étant plus nombreuses que les hommes sauf au Yukon, dans les Territoires du Nord-Ouest et au Nunavut. La population canadienne à laquelle nos résultats sont généralisables (c.-à-d. Canadiens n’habitant pas en établissement et n'étant pas membres des Forces canadiennes, etc.) a connu une croissance entre 1994-1995 et 2007-2008, passant de $18 \quad 836000$ à 24639000 individus.

\section{Douleur chronique}

$\mathrm{Au}$ cours du premier cycle (1994-1995), $18,9 \%$ (IC à $95 \%$ : 18,1 à 19,7) des Canadiens ont déclaré être atteints de douleur chronique; cette proportion est tombée à 15,1\% (IC à $95 \%$ : 14,5 à 15,7) au cours du cycle suivant (1996-1997), pour ensuite connaître une augmentation globale la portant à 18,5 \% (IC à $95 \%$ : 18,0 à 19,0) en 2007-2008. De manière générale, la différence de prévalence observée entre deux cycles consécutifs n'était pas significative. Cependant, le cycle 1996-1997 était associé à une prévalence significativement inférieure à tous les autres cycles à l'exception de 1998-1999. La figure 1 illustre la prévalence de la douleur chronique pour les années 1994-1995 à 2007-2008. 
FIGURE 4

Prévalence brute de la douleur chronique chez les Canadiennes selon les données transversales de l'Enquête nationale sur la santé de la population et de l'Enquête sur la santé dans les collectivités canadiennes, selon l'âge

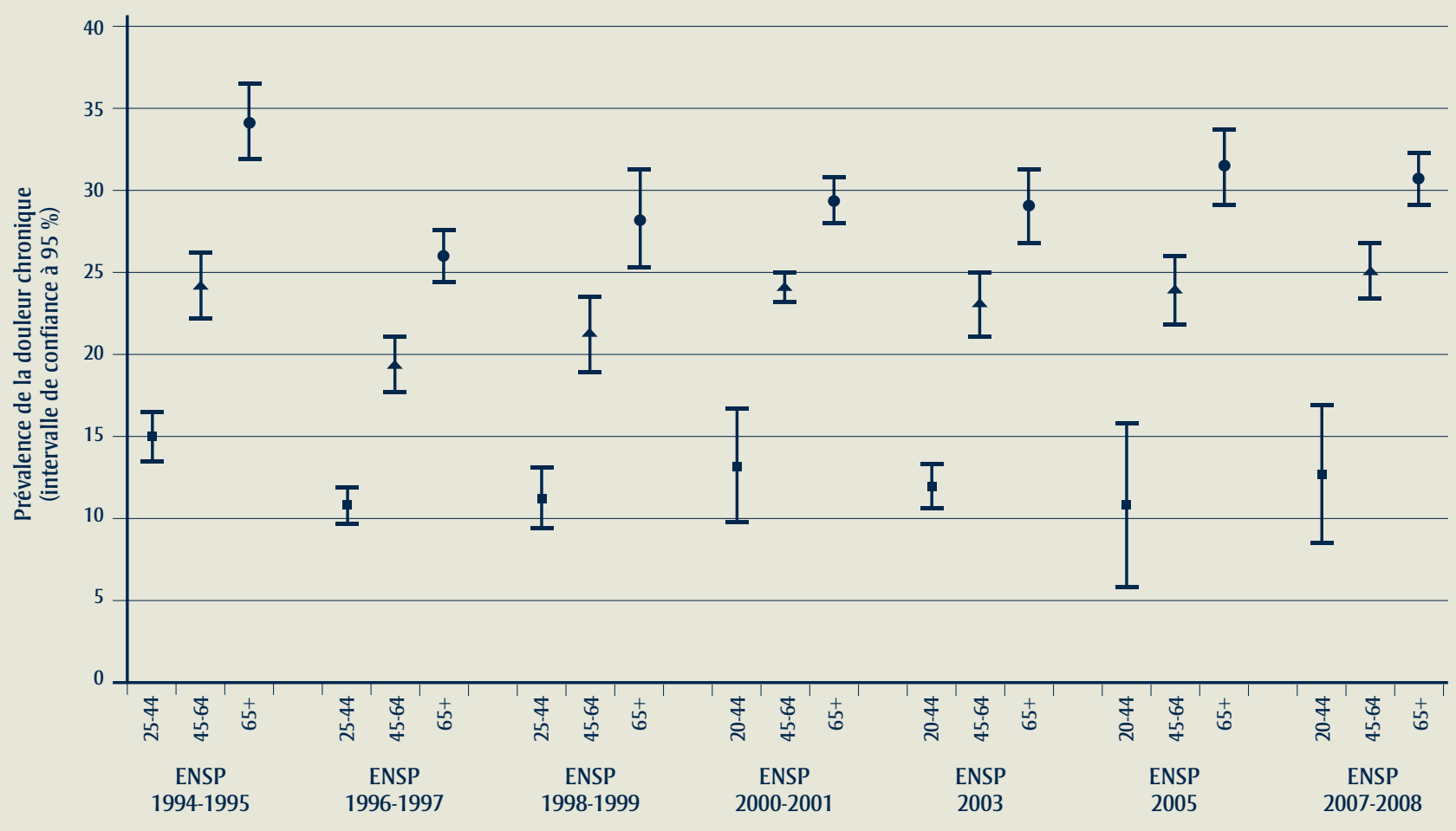

Remarque : Les participants de toutes les provinces (mais non des territoires) ont répondu aux questions sur la « douleur habituelle » à chaque ENSP. En 2000-2001 et 2007-2008, des participants de toutes les provinces et du Yukon, des Territoires du Nord-Ouest (T. N.-O.) et du Nunavut y ont répondu. En 2003, seuls les participants de la côte Est et du Québec ont répondu aux questions sur la douleur et, en 2005, seuls les participants de la Colombie-Britannique y ont répondu. Ces résultats ont cependant été pondérés en fonction de la population canadienne totale.

Pour tous les cycles à l'étude, les femmes étaient plus nombreuses à signaler des douleurs que les hommes. La prévalence de la douleur chronique s'établissait en effet entre $16,5 \%$ (IC à $95 \%: 15,6$ à 17,4 ) en $1996-1997$ et $21,5 \%$ (IC à $95 \%: 20,2$ à $22,8)$ en 1994-1995 chez les femmes contre $13,6 \%$ (IC à $95 \%: 12,5$ à 14,7) en 2003 et $16,2 \%$ (IC à $95 \%$ : 14,8 à 17,6\%) en 1994-1995 chez les hommes. Globalement, il n'y avait aucune différence significative de la prévalence de la douleur selon le sexe au fil du temps, même si des différences significatives de la prévalence étaient observées entre les sexes (figure 1).

La prévalence de la douleur variait significativement entre les groupes d'âge (figure 2). La plus forte prévalence de la douleur chronique $(23,9 \%$ à $31,3 \%$ ) était observée chez les membres du dernier groupe (65 ans et plus), mais il n'y avait pas d'évolution significative avec le temps. De manière générale, chez les hommes comme chez les femmes, il existait une différence significative de la prévalence d'un groupe d'âge à l'autre (figures 3 et 4); de plus, les femmes appartenant aux deux tranches d'âge supérieures (45-64 ans et 65 ans et plus) signalaient de la douleur chronique dans une proportion significativement plus grande que les hommes des mêmes groupes d'âge. La prévalence la plus élevée était observée pour tous les cycles chez les femmes de 65 ans et plus, et elle se situait entre 26,0\% (IC à $95 \%$ : 24,4 à 27,6 ) en 1996-1997 et 34,2 \% (IC à $95 \%: 31,9$ à 36,5$)$ en 1994-1995.

\section{Limitations fonctionnelles associées à la douleur chronique}

La majorité des répondants atteints de douleur chronique signalaient aussi des limitations fonctionnelles associées à cette douleur. En effet, la proportion de répondants ayant signalé au moins quelques activités empêchées par la douleur représentait entre $11,4 \%$ de la population totale en 1996-1997 (IC à $95 \%$ : 10,8 à 12,0 ) et $13,3 \%$ en 2000-2001 (IC à $95 \%$ : 13,0 à 13,6) et en 2007-2008 (IC à $95 \%$ : 12,8 à 13,8) (figure 5). Globalement, pour chaque cycle, davantage de femmes que d'hommes signalaient des limitations fonctionnelles associées à la douleur. Si un nombre significativement supérieur de femmes voyaient leur douleur empêcher la réalisation de quelques ou de plusieurs activités (figure 6), de manière générale, aucune différence n'était observée d'un sexe à l'autre quant à l'entrave à la plupart des activités ou à aucune d'entre elles. Finalement, il n'existait aucune différence statistiquement significative entre les années consécutives. L'évolution dans le temps de la prévalence des limitations fonctionnelles était semblable à celle de la douleur chronique.

La proportion de données manquantes sur la douleur chronique se situait d'un cycle à l'autre entre $0,1 \%$ et $0,5 \%$. 


\section{Analyse}

La présente étude est la première du genre à examiner la prévalence de la douleur chronique et des limitations fonctionnelles qui lui sont associées chez les adultes canadiens sur une période de 14 ans (1994-2008).

Sauf entre le $1^{\text {er }}$ (1994-1995) et le $2^{\text {e }}$ cycle (1996-1997), où une baisse significative a été observée (figure 1), la prévalence de la douleur chronique s'est graduellement accrue avec le temps. Même si cette évolution globale n'était pas statistiquement significative, la différence entre les cycles 1996-1997 et 2007-2008 l'était, ce qui montre une réelle augmentation de la prévalence de la douleur chronique avec le temps.

Les données estimatives sur la prévalence obtenues dans le cadre de la présente étude $(15,1 \%$ à $18,9 \%)$ s'inscrivent dans les estimations canadiennes antérieures $(11 \% \text { à } 44 \%)^{2,6-13}$. La divergence pourrait être attribuable aux différences relatives au plan d'échantillonnage, à la taille d'échantillon et à la définition de la douleur chronique employés. Par le passé, les études populationnelles réalisées auprès de grands échantillons (plus de 10000 participants) comme les nôtres ont plus souvent conclu à des prévalences estimatives inférieures (de $11 \%$ à environ $21 \%$ ) que les études comptant moins de participants $^{1,9-11,13-15,35,36}$.

Les études ayant employé une définition de la douleur chronique identique ou semblable à celle de l'ENSP et de l'ESCC ont aussi mis en évidence des prévalences estimatives (11 \% à $17 \%$ ) comparables à nos résultats ${ }^{1,8-11,13}$. Trois de ces études étaient fondées sur le cycle 1996-1997 de l'ENSP ${ }^{9,11,13}$; même si l'une d'entre elles ne tenait compte que de la douleur chronique non associée au cancer ${ }^{11}$ et qu'une autre analysait toutes les douleurs chroniques ${ }^{13}$, les estimations de la prévalence obtenues demeuraient semblables. Les études ayant employé un cadre temporel précis pour définir la douleur chronique (p. ex. 3 ou 6 mois) étaient plus sujettes à conclure à des prévalences estimatives supérieures à celles que nous avons obtenues à l'aide d'une définition plus générale (c.-à-d. douleur habituelle) 2,6,7,12 Cependant, la petite taille des échantillons analysés pourrait aussi expliquer ces résultats supérieurs ${ }^{2,6,7,12}$. Finalement, dans certains cas, il était impossible de déterminer avec certitude si les résultats sur la prévalence étaient fondés sur une mesure validée $e^{19,25,37}$. Or les études n'employant pas de définitions validées doivent être interprétées avec prudence.

La majorité des répondants ayant affirmé être atteints de douleur chronique ont aussi signalé des limitations fonctionnelles attribuables à cette douleur. Notre estimation de la prévalence de ces limitations des activités quotidiennes (11,4 \% à 13,3 \%) concorde d'ailleurs avec les résultats d'une étude australienne ayant aussi conclu à la présence de limitations des activités quotidiennes associées à la douleur chronique (femmes : 13,5\%, hommes : $11,0 \%)^{14}$.

Nous avons constaté que les femmes étaient plus nombreuses à signaler des douleurs chroniques que les hommes et que la prévalence de ces douleurs augmentait généralement avec l'âge. Ces conclusions s'appliquent à tous les cycles étudiés et concordent avec les données présentées dans la littérature $2,5,6-8,11,12,14-18,20,37$. Nous avons aussi conclu que la plus forte prévalence de la douleur chronique pouvait être observée chez les femmes les plus âgées (65 ans et plus) et que la plupart des participants atteints de douleur chronique signalaient aussi des limitations des activités associées à cette douleur; là encore, la prévalence était supérieure chez les femmes.

Une limite de notre étude tient au fait que nous n'avons pas pris en compte les maladies dont le lien avec la douleur chronique est reconnu, notamment l'arthrite, et que, par conséquent, nous n’avons pu établir de distinction entre la douleur associée à la maladie et la douleur chronique d'étiologie inconnue. Ce facteur pourrait partiellement expliquer l'observation d'une prévalence supérieure de la douleur chronique chez les femmes âgées, dont on sait qu'elles signalent davantage d'atteintes pouvant entraîner des douleurs chroniques que les hommes (p. ex. fibromyalgie, arthrite/ rhumatismes, lombalgies, migraines ${ }^{38}$. Les différences de la prévalence estimative observées à l'échelle mondiale pourraient être réelles ou encore attribuables à de nombreux facteurs dont les habitudes de vie, la répartition selon l'âge, la perception de la douleur et les traitements offerts ${ }^{36}$. C'est pourquoi il conviendrait de procéder à une étude longitudinale afin d'acquérir une meilleure connaissance des facteurs pouvant accroître le risque de douleur chronique.

Bien que les volets transversaux sur les ménages de l'ENSP et de l'ESCC soient représentatifs de la majeure partie de la population canadienne, ils excluent tous deux les personnes habitant en établissement $^{21,22}$. Ce facteur pourrait avoir conduit à une sous-estimation de la prévalence de la douleur chronique au Canada car, selon toute vraisemblance, les personnes habitant dans des résidences pour personnes âgées et des établissements de soins de longue durée seraient nombreux à souffrir de douleur chronique ${ }^{39}$. Une autre lacune de l'ENSP et de l'ESCC concerne le fait que la question portant sur la douleur ne précise pas la durée de la « douleur habituelle »; ainsi, des répondants atteints d'autres maladies pourraient aussi signaler de la douleur chronique. Cependant, nos résultats concordent avec ceux d'une étude transversale fondée sur les données de l'ENSP 1996-1997 ayant tenu compte des facteurs liés au dossier médical et à l'état de santé ${ }^{11}$, en plus d'être comparables aux résultats d'autres études ayant employé une définition générale de la douleur chronique $^{1,8-11,13}$. Finalement, bien que semblables d'une enquête à l'autre, les groupes d'âge visés par l'ENSP (25 ans et plus) et par l'ESCC (20 ans et plus) n'étaient pas identiques; les résultats obtenus pour les différents cycles étaient toutefois similaires, et, comme d'autres études ont par le passé été réalisées auprès de ces groupes d'âge ${ }^{13,24,25}$, ils pourront être comparés à la littérature. Bien sûr, la question du biais de rappel pourrait être soulevée comme il s'agissait de questionnaires auxquels les participants répondaient eux-mêmes.

Cette étude comporte plusieurs points forts dignes de mention. D'abord, les sept cycles de l'ENSP et de l'ESCC ont tous été réalisés auprès d'un important échantillon randomisé, et il y avait peu 
FIGURE 5

Prévalence brute de la douleur chronique associée aux limitations fonctionnelles selon les données transversales de l'Enquête nationale sur la santé de la population et de l'Enquête sur la santé dans les collectivités canadiennes

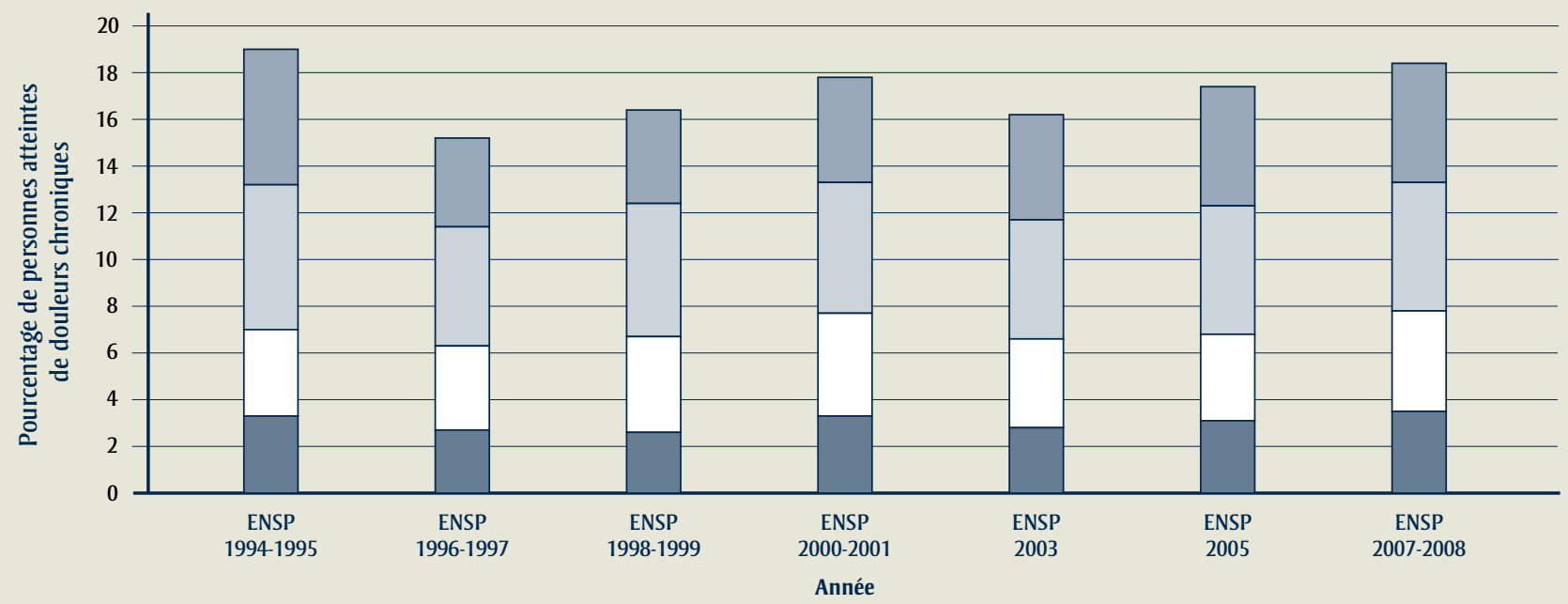

$\square$ Douleur n'entravant aucune activité
$\square$ Douleur entravant un petit nombre d'activités $\quad \square$ Douleur entravant certaines activités
*L'ENSP comprend les personnes de 25 ans et plus et l'ESCC comprend les personnes de 20 ans et plus

Nota : Les participants de toutes les provinces (mais non des territoires) ont répondu aux questions sur la « douleur habituelle » à chaque ENSP. En 2000-2001 et 2007-2008, des participants de toutes les provinces et du Yukon, des Territoires du Nord-Ouest (T. N.-O.) et du Nunavut y ont répondu. En 2003, seuls les participants de la côte Est et du Québec ont répondu aux questions sur la douleur et, en 2005, seuls les participants de la Colombie-Britannique y ont répondu. Ces résultats ont cependant été pondérés en fonction de la population canadienne totale.

\section{FIGURE 6}

Prévalence brute de la douleur chronique chez les hommes et les femmes associée aux limitations fonctionnelles selon les données transversales de l'Enquête nationale sur la santé de la population et de l'Enquête sur la santé dans les collectivités canadiennes

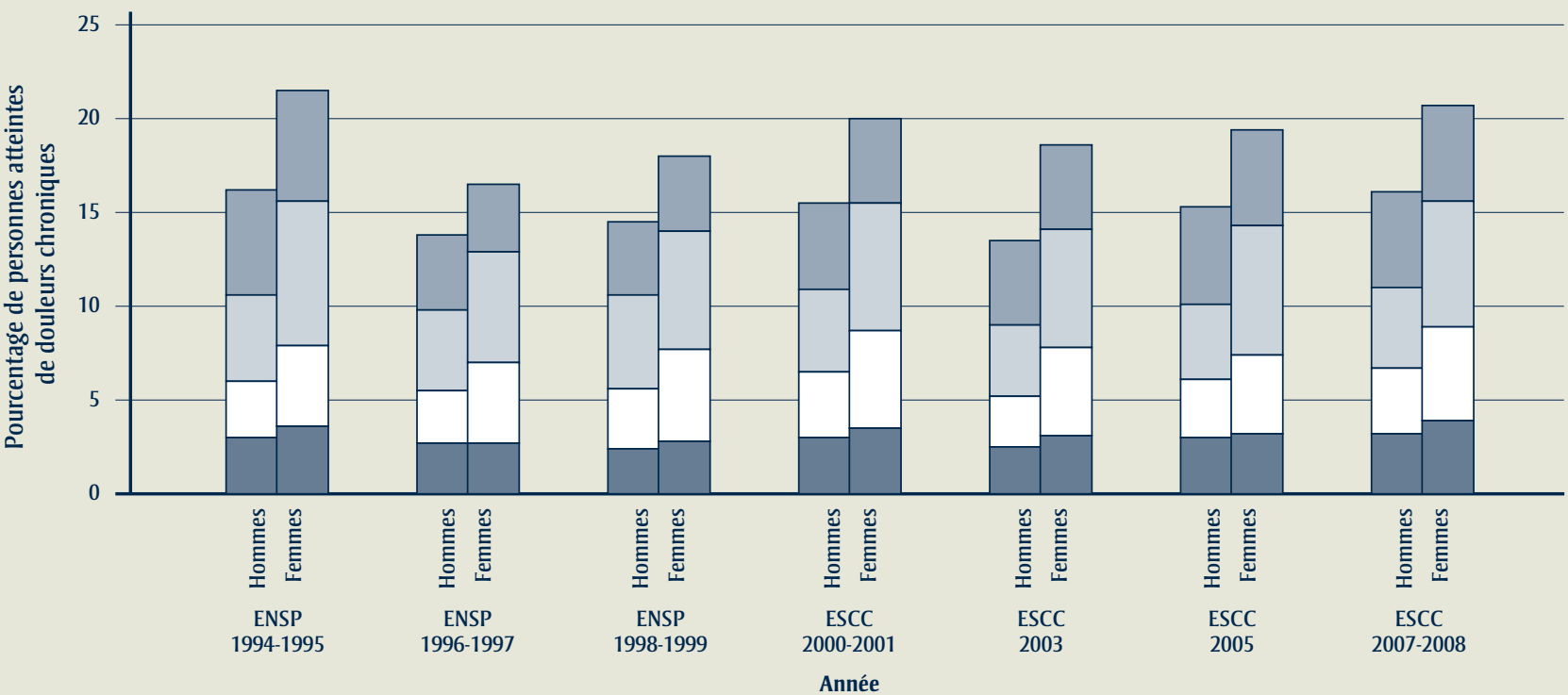

$\square$ Douleur n'entravant aucune activité $\quad \square$ Douleur entravant certaines activités

$\square$ Douleur entravant un petit nombre d'activités $\square$ Douleur entravant la plupart des activités

${ }^{*}$ L'ENSP comprend les personnes de 25 ans et plus et l'ESCC comprend les personnes de 20 ans et plus

Nota : Les participants de toutes les provinces (mais non des territoires) ont répondu aux questions sur la «douleur habituelle » à chaque ENSP. En 2000-2001 et 2007-2008, des participants de toutes les provinces et du Yukon, des Territoires du Nord-Ouest (T. N.-O.) et du Nunavut y ont répondu. En 2003, seuls les participants de la côte Est et du Québec ont répondu aux questions sur la douleur et, en 2005, seuls les participants de la Colombie-Britannique y ont répondu. Ces résultats ont cependant été pondérés en fonction de la population canadienne totale. 
de données manquantes. Une telle taille d'échantillonnage permet la généralisation des résultats à l'ensemble de la population canadienne (à l'exception des quelques groupes mentionnés précédemment); d'ailleurs, après comparaison des données du recensement canadien et des données de l'ENSP 1996-1997 par standardisation directe, Van Den Kerkhof et coll. ont conclu à la représentativité de l'échantillon et à la possibilité de généralisation des résultats à la population du Canada ${ }^{13}$. Ensuite, les questions sur la douleur de l'ENSP et de l'ESCC sont reconnues comme une mesure valide de la prévalence de la douleur chronique $^{9,13,39}$. Les résultats obtenus fournissent donc une estimation fiable et précise de la prévalence de la douleur chronique et des limitations des activités quotidiennes qui lui sont associées au Canada.

\section{Conclusion}

Cette étude est la première à analyser la prévalence de la douleur chronique au Canada sur une longue période, et elle permet de conclure que la douleur chronique est présente chez les Canadiens (prévalence de $15,1 \%$ à $18,9 \%$ ), qu'elle est plus fréquente chez les femmes (16,5\% à $21,5 \%$ ) et chez les personnes âgées $(23,9 \%$ à $31,3 \%)$ et qu'elle empêche la réalisation d'au moins quelques activités chez de nombreuses personnes atteintes $(11,4 \%$ à $13,3 \%)$. Comme les études transversales ne permettent pas de déterminer l'incidence, les prédicteurs ou les causes d'une maladie ou d'une atteinte, nous croyons qu'il s'avère nécessaire de procéder à une étude longitudinale de la douleur chronique au Canada afin de combler ces lacunes.

\section{Financement}

Nous avons bénéficié d'une bourse Freda Paltiel pour la consultation des statistiques. Nous avons aussi reçu du financement sous forme d'une bourse d'études supérieures de l'Université Queen's.

\section{Références}

1. Millar WJ. La douleur chronique. Rapports sur la santé. 1996;7(4):31 58.

2. Moulin DE, Clark AJ, Speechley M, Morley Forster PK. Chronic pain in Canada prevalence, treatment, impact and the role of opioid analgesia. Pain Res Manag. 2002 Winter;7(4):179 84

3. Pain Facts [Internet]. Edmonton (AB): Chronic Pain Association of Canada. [Consultation le 5 avril 2010]. Consultable en ligne à la page: http://www .chronicpaincanada.com

4. Andersson HI, Ejlertsson G, Leden I, Rosenberg C. Chronic pain in a geographically defined general population: studies of differences in age, gender, social class, and pain localization. Clin J Pain. 1993;9(3):174-82.

5. Elliott AM, Smith BH, Penny KI, Smith WC, Chambers WA. The epidemiology of chronic pain in the community. Lancet. 1999 Oct; 354(9186):1248-52.

6. Birse TM, Lander J. Prevalence of chronic pain. Can J Public Health. 1998 Mar-Apr;89(2):129-31.

7. Boulanger A, Clark AJ, Squire P, Cui E, Horbay GL. Chronic pain in Canada: have we improved our management of chronic noncancer pain? Pain Res Manag. 2007 Spring;12(1):39-47.

8. Crook J, Rideout E, Browne G. The prevalence of pain complaints in a general population. Pain. 1984 Mar;18(3):299-314.

9. Health Surveillance Alberta Health. Chronic pain in Alberta: a portrait from the 1996 National Population Health Survey and the 2001 Canadian Community Health Survey [Internet]. Mai 2003 [Consultation le 26 janvier 2011]. PDF téléchargeable à partir du lien : www.health.alberta.ca/ documents/Chronic-Pain-Survey-2003.pdf

10. Meana M, Cho R, DesMeules M. Chronic pain: the extra burden on Canadian women. BMC Womens Health. 2004 Aug; 4 Suppl 1:S17.
11. Rashiq S, Dick BD. Factors associated with chronic noncancer pain in the Canadian population. Pain Res Manag. 2009 Nov-Dec;14(6):454-60.

12. Tripp DA, VanDenKerkhof EG, McAlister M. Prevalence and determinants of pain and pain-related disability in urban and rural settings in southeastern Ontario. Pain Res Manag. 2006 Winter;11(4):225-33.

13. Van Den Kerkhof EG, Hopman WM, Towheed TE, Anastassiades TP, Goldstein DH; Canadian Multicentre Osteoporosis Study Research Group. The impact of sampling and measurement on the prevalence of self-reported pain in Canada. Pain Res Manag. 2003 Fall;8(3):157-63.

14. Blyth FM, March LM, Brnabic AJ, Jorm LR, Williamson M, Cousins MJ. Chronic pain in Australia: a prevalence study. Pain. 2001 Jan;89(2-3):127-34.

15. Eriksen J, Jensen MK, Sjogren P, Ekholm O, Rasmussen NK. Epidemiology of chronic non-malignant pain in Denmark. Pain. 2003 Dec;106(3):221-8.

16. Rustoen T, Wahl AK, Hanestad BR, Lerdal A, Paul S, Miaskowski C. Prevalence and characteristics of chronic pain in the general Norwegian population. Eur J Pain. 2004 Dec;8(6):555-65.

17. Sa K, Baptista AF, Matos MA, Lessa I. Prevalence of chronic pain and associated factors in the population of Salvador, Bahia. Rev Saude Publica. 2009 Aug; 43(4):622-30.

18. Catala E, Reig E, Artes M, Lopez JS, Segu JL, Aliaga L. Prevalence of pain in the Spanish population: telephone survey in 5000 homes. Eur J Pain. 2002 April;6(2):133-40.

19. Sjogren P, Ekholm O, Peuckmann V, Gronbaek M. Epidemiology of chronic pain in Denmark: an update. Eur J Pain. 2009 Mar;13(3):287-92.

20. Ng KF, Tsui SL, Chan WS. Prevalence of common chronic pain in Hong Kong adults. Clin J Pain. 2002 Sep-Oct;18(5):275-81. 
21. Statistique Canada. Enquête nationale sur la santé de la population - Volet ménages : transversal (ENSP) [Internet]. Ottawa (Ont.): Statistique Canada; 2000 [Consultation le 25 juin 2009]. Consultable en ligne à la page : http://www.statcan.gc.ca/cgi-bin/imdb /p2SV_f.pl?Function=getSurvey\&SDDS=32 $36 \&$ lang $=$ en $\& \mathrm{db}=\mathrm{imdb} \& \mathrm{adm}=8 \& \mathrm{dis}=2$

22. Statistique Canada. Enquête sur la santé dans les collectivités canadiennes (ESCC) [Internet]. Ottawa (Ont): Statistique Canada; 2009 [mise à jour le 24 juin 2009; consultation le $1^{\mathrm{er}}$ mars 2010]. Consultable en ligne à la page : http://www.statcan .gc.ca/cgi-bin/imdb/p2SV_f.pl?Function=get Survey\&SDDS $=3226 \&$ lang $=e n \& d b=i m d b \& a$ $\mathrm{dm}=8 \&$ dis $=2$

23. Division de la statistique de la santé, Statistique Canada. Enquête sur la santé dans les collectivités canadiennes, 20072008, Cycle 4.1 [Données et documentation (dossier « Guide de l'utilisateur 20072008 »] [Internet]. Ottawa (Ont.): Statistique Canada; 2009 [consultation le $1^{\text {er }}$ mars 2010]. Consultable en ligne à la page : http://search1 . odesi.ca/details/view.html?q=Canadian+ Community + Health + Survey, + 2007-2008, $+2009+$ Cycle $+4.1 \&$ field $=\&$ coll $=$ all $\&$ date-gt $=1871 \&$ date-lt $=2011 \&$ uri=/odesi/escc_82M 0013_F_2007-2008_c4.1.xml

24. Elliott AM, Smith BH, Hannaford PC, Smith WC, Chambers WA. The course of chronic pain in the community: results of a 4-year follow-up study. Pain. 2002 Sept; 99(1-2):299-307.

25. Magni G, Marchetti M, Moreschi C, Merskey H, Luchini SR. Chronic musculoskeletal pain and depressive symptoms in the National Health and Nutrition Examination. I. Epidemiologic follow-up study. Pain. 1993 May;53(2):163-8.

26. Statistique Canada. Enquête nationale sur la santé de la population 1994-1995, Guide de l'utilisateur [Internet]. Ottawa (Ont.): Statistique Canada; 1995 [page mise à jour le 7 janvier 2010; consultation le $1^{\text {er }}$ mars 2010]. Consultable en ligne à la page : http:// www.statcan.gc.ca/dli-ild/data-donees/ftp/ nphs-ensp/nphs1994-ensp1994-fra.htm
27. Statistique Canada. Enquête nationale sur la santé de la population 1996-1997, Guide de l'utilisateur, Questionnaire [Internet]. Ottawa (Ont.): Statistique Canada; 1998 [page mise à jour le 7 janvier 2010; consultation le $1^{\text {er }}$ mars 2010]. Consultable en ligne à la page : http://www.statcan.gc.ca /dli-ild/data-donnees/ftp/nphs-ensp/nphs 1996-ensp1996-fra.htm

28. Statistique Canada. Enquête nationale sur la santé de la population 1998-1999, Questionnaire, Guide de l'utilisateur [Internet]. Ottawa (Ont.): Statistique Canada; 2000 [page mise à jour le 7 janvier 2010; consultation le1 ${ }^{\text {er }}$ mars 2010]. Consultable en ligne à la page : http:// www.statcan.gc.ca/dli-ild/data-donnees /ftp/nphs-ensp/nphs1998-ensp1998-fra.htm

29. Statistique Canada. Enquête sur la santé dans les collectivités canadiennes, Cycle 1.1, Questionnaire, Manuel de codes, Guide de l'utilisateur [Internet]. Ottawa (Ont.): Statistique Canada; 2003 [page mise à jour le 7 janvier 2010; consultation le1 ${ }^{\mathrm{er}}$ mars 2010]. Consultable en ligne à la page : http://www .statcan.gc.ca/dli-ild/data-donnees/ftp/cchs -escc/cchs-escc1_1-fra.htm

30. Statistique Canada. Enquête sur la santé dans les collectivités canadiennes, Cycle 2.1, Questionnaire, Guide de l'utilisateur [Internet]. Ottawa (Ont.): Statistique Canada; 2005 [page mise à jour le 7 janvier 2010; consultation le1 ${ }^{\text {er }}$ mars 2010]. Consultable en ligne à la page : http:// www.statcan.gc.ca/dli-ild/data-donnees /ftp/cchs-escc/cchs-escc2_1-fra.htm

31. Statistique Canada. Enquête sur la santé dans les collectivités canadiennes (ESCC) - Cycle 3.1 (2005) - Fichier de microdonnées à grande diffusion (FMGD) - Guide de l'utilisateur [Internet]. Ottawa (Ont.): Statistique Canada; 2006 [consultation le $1^{\text {er }}$ mars 2010]. Consultable en ligne à la page : http://www.statcan.gc.ca/dli-ild /meta/cchs-escc/cycle3-1/guide-fra.pdf
32. Statistique Canada. Enquête sur la santé dans les collectivités canadiennes - Cycle 3.1 - Questionnaire final [Internet]. 2006 [consultation le 20 avril 2011]. PDF téléchargeable à partir du lien : http://www.statcan. gc.ca/imdb-bmdi/instrument/3226_Q1_V3fra.pdf

33. Statistique Canada. Enquête sur la santé dans les collectivités canadiennes (ESCC) - Questionnaire de 2007 [Internet]. 2007 [consultation le 20 avril 2011]. PDF téléchargeable à partir du lien : http:// www.statcan.gc.ca/imdb-bmdi/instrument /3226_Q1_V4-fra.pdf

34. SPSS Inc. IBM SPSS Bootstrapping 19: Part 1: Users Guide: Bootstrapping [Internet]. 2010 [consultation le 20 avril 2011]. PDF téléchargeable à partir du lien : http:// support.spss.com/productsext/statistics /documentation/19/client/User $\% 20$ Manuals/English/IBM\%20SPSS \% 20 Bootstrapping \% 2019.pdf

35. Hardt J, Jacobsen C, Goldberg J, Nickel R, Buchwald D. Prevalence of chronic pain in a representative sample in the United States. 2008; Pain Med. 2008 Oct;9(7):803-12.

36. Breivik H, Collett B, Ventafridda V, Cohen R, Gallacher D. Survey of chronic pain in Europe: prevalence, impact on daily life, and treatment. Eurp J Pain. 2006 May;10(4):287-333.

37. Von Korff M, Dworkin SF, Le Resche L. Graded chronic pain status: an epidemiologic evaluation. Pain. 1990 Mar;40(3):279-91.

38. Munce SE, Stewart DE. Gender differences in depression and chronic pain conditions in a national epidemiologic survey. Psychosomatics. 2007 Sep-Oct;48(5): 394-9.

39. Statistique Canada. Enquête nationale sur la santé de la population - Volet établissements de soins de santé, longitudinal (ENSP) [Internet]. 2000 [consultation le 29 avril 2011] Consultable en ligne à la page : http:// www.statcan.gc.ca/cgi-bin/imdb/p2SV_f .pl? Function $=$ getSurvey $\&$ SurvId $=19819 \&$ Su rvVer $=1 \&$ InstaId $=20083 \&$ InstaVer $=2 \&$ SDDS $=5003 \&$ lang $=e n \& d b=I M D B \& a d m=8 \&$ dis $=2$ 